\title{
Pratiques
}

Linguistique, littérature, didactique

\section{Un lieu de tension entre posture de lecteur et posture de correcteur : les traces des enseignants de français sur les copies des élèves}

Jean-Luc Pilorgé

\author{
(2) OpenEdition \\ Journals \\ Édition électronique \\ URL : http://journals.openedition.org/pratiques/1513 \\ DOI : 10.4000/pratiques. 1513 \\ ISSN : 2425-2042 \\ Éditeur \\ Centre de recherche sur les médiations (CREM)
}

Édition imprimée

Date de publication : 15 juin 2010

Pagination : 85-103

Référence électronique

Jean-Luc Pilorgé, «Un lieu de tension entre posture de lecteur et posture de correcteur : les traces des enseignants de français sur les copies des élèves », Pratiques [En ligne], 145-146 | 2010, mis en ligne le 15 juin 2010, consulté le 10 décembre 2020. URL : http://journals.openedition.org/pratiques/1513 ; DOI : https://doi.org/10.4000/pratiques.1513 


\title{
Un lieu de tension entre posture de lecteur et posture de correcteur : les traces des enseignants de français sur les copies des élèves
}

\author{
Jean-Luc Pilorgé \\ IUFM de Bretagne, école interne de I'Université de Bretagne \\ Occidentale
}

Comment l'enseignant de français aborde-t-il la lecture d'un texte d'élève de collège? Quels sont les paramètres en jeu dans le rôle très particulier qui lui est dévolu et qui l'oblige à osciller entre deux postures, celle du lecteur et celle du correcteur?

La recherche présentée étudie la tension entre ces deux attitudes, tension qui s'inscrit au cœur d'une activité ordinaire du professeur de français au collège : la correction des travaux d'écriture fictionnelle réalisés par les élèves. Cette activité quasi quotidienne se traduit par des conduites correctives très diverses mais peu interrogées par les acteurs eux-mêmes. L'enseignant adopte alors une attitude de lecture spécifique qui hésite entre une recherche d'objectivité, répondant ainsi à la déontologie minimale du correcteur, et l'implication subjective fondatrice de l'acte de lecture lui-même.

L'analyse du métadiscours qui apparaît sur les copies d'élèves sous forme d'annotations peut nous permettre de mieux comprendre les variations qui affectent les pratiques de correction. Les postures de correction adoptées de manière spontanée renvoient en effet à des représentations variées et peu objectivées de l'activité de correction et du dialogue qui s'instaure entre l'enseignant - lecteur et l'élève - scripteur. Nous cherchons donc à décrire, derrière la routine du geste de lecture de copies, la complexité des comportements. L'analyse s'intéresse aux « traces de correction », aux annotations des professeurs mais aussi à la réécriture de textes d'élèves par ces mêmes professeurs. Les dispositifs expérimentaux mis en œuvre permettent ainsi de proposer une typologie des postures adoptées par les correcteurs et tentent de cerner quelques caractéristiques du «texte attendu » par les enseignants en réponse à une consigne d'écriture.

Il s'agit finalement de mieux connaître la réalité d'une tâche professionnelle spécifique où subjectivité et normes sont à l'œuvre et restent le plus souvent implicites. 


\section{Quelques points d'appui}

Le développement des théories de la lecture a largement renouvelé les conceptions de la lecture, en particulier littéraire ${ }^{(1)}$. Si le sens d'un texte n' existe que pour et par sa lecture, les opérations qu'effectue le lecteur importent autant que le texte proprement dit qui n'existe que grâce à son concours. Que penser alors de la singularité de l'enseignant aux prises avec l'impératif de la correction des copies de ses élèves ? Comment met-il en jeu l'implication complexe réclamée par l'activité de lecture ?

Les recherches sur l'écriture, en particulier les considérations développées par la critique génétique, montrent par ailleurs combien l'acte d'écriture échappe aux représentations d'un écrit qui, d'abord bien conçu, peut être clairement énoncé par le scripteur. Comment apprécier la nature des éventuels dysfonctionnements du texte que le correcteur a sous les yeux ? Si l'intérêt porté à la correction de copies invite à s'intéresser à la fois au statut du texte à corriger et au travail de l'enseignant, on ne peut oublier les enjeux de cette pratique professionnelle singulière. Quel est le sens et l'utilité de l'évaluation des écrits des élèves?

«Le sens et l'utilité d'un apprentissage de la littérature se détermine selon la valeur qu'on leur attribue et il est difficile de séparer l'évaluation, instrument de mesure de la valeur attribuée à l'apprentissage, et la question de son sens et de son utilité - variable au demeurant selon les acteurs concernés... Or attribuer une valeur à un objet d'apprentissage, c'est faire état d'un système de valeurs et il n'est pas exagéré de dire que, sur ce point, aucun consensus ne se dessine. »" ${ }^{(2)}$

Les propos de B. Daunay pourraient fort bien s'appliquer aux apprentissages relatifs à l'écriture, surtout si l'on se limite aux écrits fictionnels. Le consensus en matière d'évaluation est difficile à construire et la pratique de correction doit être interrogée sous l'angle du rapport singulier établi entre un lecteur et un scripteur. Le lecteur empirique, sans abdiquer sa liberté fondamentale, accepte en principe de se livrer à une activité de collaboration. Tout se passe comme s'il voulait qu'on tienne compte de lui comme sujet actif capable d'effectuer les inférences nécessaires à la compréhension et de développer des stratégies d'interprétation. La participation du lecteur suppose aussi, quand il s'agit de lecture littéraire et spécifiquement de lecture de textes fictionnels, une adhésion à l'univers de référence construit par l'auteur. Un ressort essentiel $\mathrm{du}$ « bon texte » fictionnel pourrait bien être alors un état de densité de l'écriture dont le principe d'économie apparente est dicté par la nécessité de laisser au lecteur la possibilité de construire des inférences. Ces questions prennent un relief particulier lorsque l'on se préoccupe non seulement de textes légitimés mais de textes produits en situation scolaire. Comment procède alors l'enseignant?

Force est de constater qu'en cette matière, rares sont les ressources institutionnelles. Les textes officiels s'intéressent au compte-rendu collectif des travaux d'écriture plutôt qu'à l'activité de correction proprement dite. Le professeur reste une abstraction, ses réactions de lecteur et de correcteur sont à peine évoquées. Par ailleurs, la recherche en didactique de l'écriture qui a privilégié la connaissance des processus en jeu dans l'écriture des élèves et l'examen des textes qu'il s'agisse de

(1) Rouxel, A. et LANGlade, G. (dir.) Le sujet lecteur, lecture subjective et enseignement de la littérature, Actes du colloque «Sujets lecteurs et enseignement de la littérature », Presses Universitaires de Rennes, 2004.

(2) DAUnAY, B. «Comment la recherche didactique évalue les pratiques d'enseignement de la littérature ", in Enseigner et apprendre la littérature aujourd'hui, pour quoi faire ?, J.L. Dufays éditeur, Presses Universitaires de Louvain, 2007. 
«brouillons » ou de « produits finis » s'efforce de neutraliser au mieux l'activité du correcteur.

Le non-dit théorique et institutionnel correspond d'ailleurs assez bien au silence des praticiens. Les réflexions épisodiques sur l'évaluation des écrits et la recherche d'une homogénéisation des pratiques de correction ne garantissent nullement le consensus en matière de correction effective. La question est pourtant d'importance, si l'on veut bien considérer les conséquences qu'un tel non-dit peut avoir sur le rapport à l'écriture qui se construit dans les classes et chez chaque élève.

Il apparaît ainsi utile d'interroger la correction de copies comme geste professionnel, concept dont A. Jorro ${ }^{(3)}$ souligne plusieurs aspects : il a un sens pour celui qui le mobilise, une valeur symbolique, et s'appuie sur une intentionnalité en direction de celui qui le reçoit, attendant un effet ou une réaction. Nous privilégions de ce point de vue la manière dont l'enseignant prend le texte de l'élève en considération et le constitue en objet de discours pour analyser les postures mobilisées à cette occasion.

\section{Le recueil et l'analyse des « traces » de correction}

L'objectif essentiel de notre recherche est d'interroger la lecture que des enseignants mettent en œuvre lorsqu'ils corrigent des écrits d'élèves. Elle est évidemment tout à fait particulière et bien éloignée de celle que les mêmes enseignants ont l'occasion de déployer par ailleurs. Nous pouvons penser que les « postures de correcteur », spécifiquement professionnelles, se construisent en tension par rapport aux postures d'un lecteur ordinaire. Cherchant à décrire avant d'expliquer, nous avons cherché à croiser plusieurs expérimentations afin de proposer des éléments de théorisation et de dégager des invariants du geste professionnel exploré.

Ce choix supposait de recueillir des copies d'élèves corrigées par des professeurs. Il était aussi indispensable pour mener une réflexion comparative sur les attitudes de correction de maîtriser au mieux leurs conditions d'émergence et donc d'imaginer des dispositifs adaptés. Des sessions de formation, à l'intention de professeurs stagiaires ou de professeurs en formation continue, nous ont offert l'opportunité de proposer des copies identiques à des groupes de professeurs et de recueillir ainsi des corpus conséquents de corrections comparables.

Nous avons en effet fait l'hypothèse que le discours tenu, à propos des devoirs d'élèves et de la correction de copies, ne permettait pas de faire un état des lieux des pratiques et que seule l'observation contrôlée d'une pratique effective de correction pouvait répondre à notre quête d'informations. Cette stratégie impliquait des sélections complémentaires, nous avons choisi de neutraliser plusieurs paramètres qui risquaient de parasiter les résultats : le niveau d'enseignement et le type d'écrits considéré. Les productions retenues, limitées à des écrits fictionnels, concernent le seuil collège-lycée. Les textes d'élèves soumis aux corrections multiples n'ont pas de caractère exceptionnel, ni dans un sens, ni dans un autre ; nous voulions éviter toute confusion entre notre réflexion et des considérations sur l'excellence ou la déficience scolaire.

Les dispositifs conçus nous ont permis de travailler sur un échantillon opératoire de métadiscours de correcteurs, en l'occurrence les traces laissées par les professeurs sous forme d'annotations. Bien sûr, un texte tend à générer des annotations singulières, c'est pourquoi nous avons renouvelé l'expérimentation pour éviter des inférences abusives à partir de constats conjoncturels. Sans nier l'importance des déterminations personnelles des correcteurs, il nous semble ainsi possible de pro-

(3) Jorro, A., Professionnaliser le métier d'enseignant, Paris : E.S.F., 2002. 
poser les éléments d'une typologie des postures de correction que les professeurs adoptent de manière spontanée, probablement en référence à un " texte attendu », virtuel, que nous avons voulu « réaliser» en soumettant ensuite les correcteurs à l'épreuve de la réécriture d'un texte d'élève.

L'analyse détaillée des données recueillies grâce à ces divers dispositifs nous permet de mieux comprendre un geste professionnel peu interrogé, et de mettre en évidence des questions qui vont, semble-t-il, au-delà de la tâche de correction dont s'acquitte régulièrement le professeur de français. Les traces laissées sur les copies expriment des jugements émis sur des productions écrites singulières et, de plus, elles nous informent sur les manières d'envisager la discipline et le rapport aux élèves.

Nous avons pu ainsi constituer des corpus de corrections de différentes copies auprès de plusieurs groupes de professeurs ${ }^{(4)}$.

Le Métro de l'Enfer (copie M), servira de support à la mise en évidence de constats caractéristiques de l'instabilité des critères de correction. La consigne d'écriture, relativement libre, de ce premier jet demandait la rédaction d'une « nouvelle à chute surprenante ${ }^{(5)}$. La deuxième copie, La lettre d'Antigone (copie A), que nous exploitons dans la suite de l'article, répond à une commande plus contrainte, proche de ce qui est demandé dans le cadre de l'écriture d'invention. Il s'agit d'une lettre fictive de l'héroïne emprisonnée à son oncle Créon ponctuant l'étude de l'Antigone de Jean Anouilh. Le choix de ce second texte est dicté par la volonté neutraliser l' " effet-qualité » du texte à corriger. Des travaux préliminaires, que nous ne pouvons détailler ici, ont en effet permis de retenir ce texte comme «réussi » de l'avis de l'ensemble des participants.

\section{Copie M : «Le Métro de l'Enfer»}

Jeannot attendait le train. Il y avait beaucoup de monde sur le quai. Des personnes pleuraient ou rigolaient, la joie ou la peur de la vitesse, peut-être.

Des jets de fumées s'échappaient de la locomotive.

C'était impressionnant!

Enfin, le métro arriva. Les gens se bousculaient pour savoir qui passerait le premier. Moi, le bruit, la foule et tout le reste, ça me stresse.

Je passai entre les personnes qui, elles, me hurlaient aux oreilles, tout en me postillonnant au visage. Je m'assis et pris mon journal. Le train démarra.

Dix minutes passèrent, le wagon s'arrêta brusquement dans un immense tunnel sombre, la peur et les cris des enfants donnaient un sentiment de frayeur.

Et voilà que le train de l'enfer repris son élan, j'étais soulagé. Une voix se fit entendre, c'était le conducteur qui nous annonçait la panne de la locomotive, mais que tout le monde se rassure, rien de grave.

Une demie-heure plus tard, j'arrivais enfin a la gare.

Des enfants couraient dans tous les sens, et dégustaient leur barbe à papa. Des magasins de sucreries et de galettes aux chocolats étaient installés dans tous les recoins de la ville. Une foule incroyable attendait devant les jeux de vitesses où encore devant la chenille pour les plus petits.

Moi, le train de l'enfer, ça me suffit, je préfère rentrer chez moi et lire mon journal tranquillement.

(4) Les données recueillies permettent de réfléchir à des corrections multiples d'une même copie effectuées dans une situation contrôlée : tous les participants bénéficient des mêmes informations. L'ensemble des annotations produites par les correcteurs sur chacune des productions d'élèves ont été ensuite répertoriées et analysées.

(5) Copie M et copie A : les deux textes retenus sont des productions d'élèves en classe de troisième. 


\section{Cpéon,}

Vous m'avez. Fa'te paisannieke. je ne dons plus, ne mange plus, c'est à peine s' j'ar une. âme ici, mais ce n'est pas votme doósía la cause de cola ... Non, êthe en ca ge mimparte per, ce qui me met dans cet fíat, "grand" ceéon, c'est

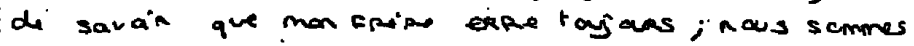
au mime sang, je me dois de le caire rejaindre les siers, daisses-mai an prizon, je mien ricte. Ila jene poux aien. faite, appoat peut Tetpa joires auec vate colpabilité...

Pdynite est votre naver, it niekart pas parrant certes, mais, vars aussi, partages \& sang qu' ne carle plis dins ses: veines. que disa-ton de The'bes dans su siectes al venia? qu'elle est len ville oi lis princes réclimants implement teun du niont pas ke drait a'un vie. ajpec's la mest. Qu'on tenisse un cadavte plein. de verpeis a'lea ive it a' l'oderat d trous, même des phis jeúnes. que son Ra aurrera's nibtair qu'in lecke qui a. dit "avi"... vous ne vous préroccupez donc pas. de ce que dira le manda? Il est indigat, diun prérender Rai, de lasssen pourrix sen reuveu, d'en taipe in hasinge

pova saristaire la ville.:.

De quai ave 3 -vas peen? Parquoi ne pas prondre cette dezision de eibereer mon freine, quivas.

cane äs yeux"? Un käche ai, was eres uncacre er ti.seianesis. vous punira.

Si Polynice avair pegré sukthe'bes or

que vas ékilez moat en rpairae pendant sain beigre. lvi, aupait e le caan de vas a pardonnes quirte a'deçeveris a fouple ... Main's vaus many de trat, ole cean, d'odace, de pirié ett méme de

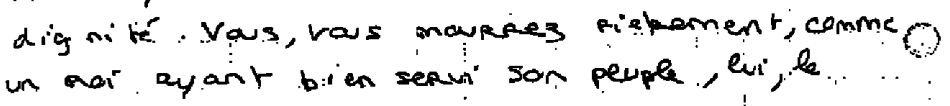
meprita:! auss:!

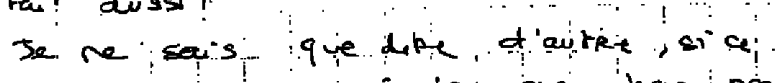
nest experimer mon prepris pas vatere posonne

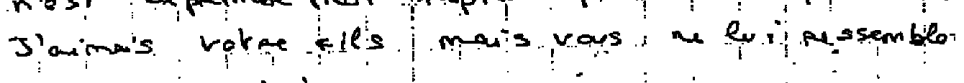

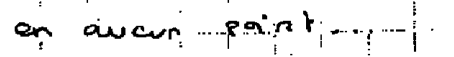




\section{De la convergence des critères aux divergences d'application :}

\section{la place à la subjectivité}

Notre propos n'est pas de revenir sur des considérations docimologiques déjà explorées et qui ont montré les écarts de notation qui peuvent exister d'un correcteur à l'autre. L'examen systématique des annotations doit nous renseigner plus avant sur les motivations des interventions correctives. Quelle conception du texte et du devoir d'élève est à l'œuvre chez les enseignants de français ? Quelle représentation de la discipline et du rôle de l'enseignant s'exprime dans ce geste professionnel ordinaire?

L'analyse des annotations des correcteurs du Métro de l'Enfer autorise un certain nombre de constats qu' on pourra juger surprenants. La consigne d'écriture donnée à la classe imposait aux élèves de s'inscrire dans un cadre générique contraignant sur lequel la plupart des correcteurs de notre échantillon se sont exprimés. Les annotations concernant les caractéristiques génériques ont été classées selon l'appréciation formulée en trois ensembles sensiblement égaux dont on trouvera ci-dessous quelques exemples significatifs.

\begin{tabular}{|l|l|l|}
\hline $\begin{array}{l}\text { La nouvelle et/ou la chute } \\
\text { est/sont positivement } \\
\text { appréciée(s) }\end{array}$ & $\begin{array}{l}\text { La présence d'une chute est } \\
\text { reconnue mais appréciée } \\
\text { négativement ou soumise } \\
\text { à forte critique }\end{array}$ & $\begin{array}{l}\text { Le non respect du genre } \\
\text { et/ou l'absence de chute } \\
\text { est/sont noté(e)(s) }\end{array}$ \\
\hline $\begin{array}{l}\text { Bon respect de la technique de } \\
\text { la nouvelle (chute finale) et in- } \\
\text { ventivité judicieuse. / Chute : } \\
\text { TB }\end{array}$ & $\begin{array}{l}\text { Intérêt de la chute ? Où est le } \\
\text { rapport avec la description de } \\
\text { la ville qui précède? }\end{array}$ & $\begin{array}{l}\text { Mais le principe de la nouvelle } \\
\text { n'est pas bien compris : il te } \\
\text { faut une chute, soit une fin } \\
\text { surprenante. }\end{array}$ \\
\hline $\begin{array}{l}\text { Ta chute est particulièrement } \\
\text { bien travaillée : le reste du } \\
\text { texte donnait des indices mais } \\
\text { sans laisser présager de la fin : } \\
\text { tu crées une vraie surprise ! }\end{array}$ & $\begin{array}{l}\text { Ta chute crée-t-elle un effet de } \\
\text { surprise? }\end{array}$ & $\begin{array}{l}\text { le mécanisme de la nouvelle } \\
\text { n'est pas compris : il fallait in- } \\
\text { venter une histoire où la chute } \\
\text { devait étonner, déstabiliser le } \\
\text { lecteur. / Où est la surprise } \\
\text { créée par la chute? }\end{array}$ \\
\hline
\end{tabular}

L'extrême diversité des appréciations est remarquable : les participants n'ont pas la même conception d'une «nouvelle à chute surprenante». Les professeurs sont-ils en désaccord sur la définition? C'est difficile à envisager compte tenu du savoir partagé attendu d'une telle collectivité ${ }^{(6)}$. Même s'il y a accord tacite sur les définitions, il n'y a pas consensus sur leur application à l'objet concret que constitue le devoir d'élève. L'hésitation générique, constitutive des savoirs de référence de la discipline, garde apparemment toute son actualité même lorsqu'il s'agit d'apprécier une production scolaire. Si l'accord sur les notions existe dans l'abstraction, il ne résiste pas à la confrontation au texte réel. L'appréciation de la présence d'indices laissant présager la chute n'est pas plus consensuelle. Où l'un des correcteurs réclame la surprise, l'autre l'apprécie ! Chacun semble avoir une conception singulière du degré d'implicite nécessaire ou acceptable.

La subjectivité ne peut s'effacer lors de la mise en œuvre effective d'une lecture, même lorsque la responsabilité de correcteur filtre ses réactions.

(6) Lors de séances préalables en formation, un travail collectif de construction de séquences d'enseignement prenant appui sur des lectures de nouvelles avait permis aux stagiaires de confronter leurs points de vue et de se construire des références communes. 
Le Métro de l'Enfer posait d'autres problèmes et le classement des traces de correction a permis d'isoler plusieurs champs d'interventions marqués par un nombre significatif d'annotations. La mise en évidence d'une rupture énonciative forte et pénalisante pour l'appréciation du texte était attendue. On peut souscrire aux interventions des correcteurs qui demandent à l'élève d'homogénéiser le système énonciatif, de choisir entre $1^{\text {re }}$ et $3^{\mathrm{e}}$ personne. Les approches sont néanmoins différentes d'un correcteur à l'autre. Le choix d'un système de référence pour signaler la défaillance peut s'expliquer par un souci d'interaction pédagogique, par la volonté de voir le métadiscours effectivement compris par l'élève. Le mot «énonciation » apparaît une seule fois (Copie M16 : attention à l'énonciation), la plupart des correcteurs s'interrogent sur le choix du narrateur, attirent l'attention sur le système des personnages. La modalité interrogative peut traduire l'amorce d'un dialogue avec l'élève à moins qu'elle ne mime simplement la réaction du lecteur face au texte; en effet, les interrogatives sont rarement suivies d'injonctions de réécriture et introduisent souvent des constats déceptifs (Copie M10 : Il aurait fallu...). Le texte est défaillant par rapport à la représentation que le lecteur se fait d'une production réussie, mais ce premier jet n'est pas considéré comme un objet en devenir qui peut porter en germe les outils de son amélioration, la motivation d'une réécriture ${ }^{(7)}$.

Le correcteur signale les défaillances et peine à repérer les potentialités. De manière générale, la lecture du devoir scolaire semble se faire en référence à une norme implicite qui pose la simplicité comme valeur indépassable.

La cohérence du texte est souvent appréciée à travers la référence au moyen de transport - train ou métro - dont la confusion fait parfois écran à tout autre élément constitutif du récit. Certains correcteurs restent focalisés sur les choix lexicaux et syntaxiques (Copie M20 : D'une façon générale ton récit n'est pas assez organisé : il manque par exemple des connecteurs logiques. / Attention à ne pas sombrer dans le registre familier). Les corrections déviantes, ne soulignant pas l'insuffisante clarté du système énonciatif, sont révélatrices de la subjectivité inhérente à l'acte de correction. L'énonciation est oubliée parce qu'elle est occultée par des préoccupations plus fortes. Nombre de correcteurs sont accaparés par une correction attentive au détail et pilotée par des considérations à propos du niveau de langage par exemple.

Dans des champs en principe plus stabilisés, le désordre est aussi patent : il en va ainsi des annotations concernant le système verbal. On corrige en référence soit au fonctionnement, temps et aspects, soit aux problèmes posés par la morphologie. C'est l'un ou l'autre, jamais les deux. De surcroît, les traces correctives ne renvoient jamais à la possibilité d'un choix du scripteur mais évoquent uniquement un univers de contraintes. La forme verbale choisie est appréciée ou critiquée, parfois en vertu de règles dont on voudrait connaître l'origine (Copie M25:1. 14 «j'arrivais enfin à la gare : temps : tu confonds imparfait et passé simple ici »/Copie M6 : 1.19 " il faut garder le même temps tout au long du texte »). Lorsqu' on isole les annotations qui concernent la langue, la tendance à l'hypernormativité s'exprime avec force dans des affirmations dont la pertinence est parfois ténue.

Il ne s'agit pas de mettre en cause les compétences des correcteurs de notre échantillon mais d'explorer les mécanismes à l'œuvre dans une activité fortement automatisée. De toute évidence, les divergences sont importantes.

(7) En effet, si l'on veut bien admettre que l'erreur n'est pas celle que l'évidence nous impose, on peut aussi envisager que la rupture énonciative fait ici apparaître non pas un mais deux personnages : «Je » narrateur et protagoniste malgré lui de cette sortie foraine et « Jeannot ». L'amélioration du texte passerait alors moins par une fusion Jeannot / narrateur que par la gestion de deux personnages. Seul un correcteur a tenu compte de cette possibilité. 


\section{Les « choix » du correcteur ? une affaire de posture}

Les expérimentations menées montrent que l'activité de correction, pratique de routine, connaît des inflexions qui ont à voir avec les représentations de la tâche et plus profondément sans doute avec la conception même du métier et de l'écriture.

Les traces de correction, d'une relative homogénéité formelle, sont soumises à de notables variations qui témoignent ${ }^{(8)}$ de la diversité des attitudes adoptées. La nature précise des opérations intellectuelles mises en œuvre par l'enseignant lors de la lecture de travaux d'élèves est sans doute fort complexe mais le contenu explicite du métadiscours évaluatif nous permet d'inférer quelques caractéristiques relatives à la manière dont le correcteur envisage plus ou moins consciemment son rôle. Il n'est en effet pas certain que le professeur ait une conscience permanente de son « attitude » de correction. La situation ordinaire est peu propice aux longues réflexions et échappe rarement à une certaine routinisation des interventions; s'il est vraisemblable qu'un temps de réflexion préalable de l'enseignant permet l'explicitation consciente, au moins pour lui-même de critères de correction, cette réflexion se trouve ensuite confrontée à la diversité des copies successives et exige l'automatisation de certaines traces et de la manière de les concevoir.

La définition que propose D. Bucheton de la "posture de lecture » comme « schème pré-construit d'actions intellectuelles et langagières que le sujet convoque en réponse à une situation ou à une tâche données » ${ }^{(9)}$ peut nous aider à cerner la diversité des orientations de correction. Le mot « posture » est utilisé dans une étude portant sur la variété des lectures d'élèves de collège et il est tentant de reprendre ici ce terme pour décrire les variations qui affectent la lecture des enseignants ${ }^{(10)}$. La manière dont le terme est associé à une tâche scolaire - ce sont des écritures de commentaire produites par des élèves qui sont analysées et fondent le recours à la notion de posture - peut être transposée à la tâche professionnelle du correcteur.

Les « postures » du correcteur sont relatives à la fois au texte à lire, à la réflexion sur le contexte scolaire d'écriture, à la stratégie didactique adoptée. On peut penser que les interventions sur les copies portent la trace des motivations profondes du scripteur même si chaque texte d'élève induit une lecture et des annotations spécifiques. Le mot « posture » incite par ailleurs à envisager la possibilité de « changements de posture » : il n'est pas question de tendre à la définition de profils intangibles selon les caractéristiques personnelles des correcteurs, on préfèrera s'engager dans une tentative de modélisation étroitement dépendante de la tâche à réaliser en contexte et qui tienne compte d'une conception de la lecture comme interprétation. Même si les « dé-lires » propres à l'acte individuel et solitaire de lecture sont probablement limités dans le cadre professionnel, il n'y a pas de raison de penser que les phénomènes de résistance au texte disparaissent subitement.

Les analyses de corrections montrent qu'une production d'élève donne lieu à des

(8) Il faut garder à l'esprit la situation, théoriquement dialogique, dans laquelle se trouvent les enseignants lors de cette tâche professionnelle et ne pas minimiser la dimension pédagogique du discours tenu. Les traces qui témoignent de la lecture sont à la fois une réponse au texte soumis à correction et la marque d'un échange à des fins d'apprentissage et donc dans la perspective d'une progression des capacités scripturales de l'élève.

(9) BuCheton, D. (2000), «Les postures de lecture des élèves au collège », in Enseigner la littérature, Actes du colloque «Enjeux didactiques des théories du texte dans l'enseignement du français », Delagrave CRDP Midi-Pyrénées, pp. 201-213.

(10) Il ne s'agit pas de céder au plaisir d'une analogie qui laisserait penser que les enseignants écrivent ce qui leur passe par la tête sur les copies, mais de souligner la complexité d'une tâche professionnelle dont tous les paramètres ne sont pas nécessairement contrôlés en permanence par les acteurs. 
interprétations variées voire contradictoires, pourtant les conditions contrôlées d'expérimentation laissent peu de place aux variables contextuelles et ce sont bien les lectures des sujets qui doivent être considérées. Elles ne sont pas totalement singulières : les lecteurs respectent ce qu'ils connaissent des conditions de la production scolaire, des consignes d'écriture, des programmes d'enseignement. Ils s'inscrivent aussi dans une situation de communication spécifique envisageant, abstraitement sans doute mais parfois de manière explicite, un destinataire susceptible de comprendre le jugement porté sur sa production et d'en tirer des conséquences. L'impact de la tradition corrective apparaît dans la partition des annotations : des annotations en marge, souvent lapidaires et abréviées sont complétées par un texte bref chargé d'axiologiques, une annotation générale. Si les enseignants s'inscrivent spontanément dans ce modèle sans en contester la pertinence, marquant ainsi leur appartenance professionnelle, le contenu des interventions montre à quel point la singularité des lectures reste importante.

\section{Les postures du correcteur}

Derrière l'abstraction du lecteur de copies apparaissent des sujets lecteurs fort différents dans leur manière d'utiliser le métadiscours de correction. L'analyse des annotations permet de proposer cinq postures caractéristiques de la variété des attitudes ${ }^{(11)}$ adoptées.

\section{Le "gardien du code"}

Nous nommons ainsi la posture adoptée par le professeur qui s'intéresse à la réalisation scripturale de l'élève du point de vue de la conformité au code. Le correcteur agit sans implication notable, son point de vue de lecteur collaboratif sur le texte reste peu exprimé. Il se projette dans un rôle uniquement technique qui, dans l'univers social, le rapprocherait du correcteur typographe. Il corrige le texte en privilégiant le niveau de surface (ponctuation, mise en paragraphes, orthographe principalement) pour le rendre conforme à un modèle qui n'est pas envisagé comme devant être explicité. Celui-ci est-il clairement construit? Rien n'est moins sûr. Si la correction orthographique renvoie à des règles précises ${ }^{(12)}$ sur lesquelles les lecteurs experts peuvent s'entendre, on constate que la mise en paragraphes, l'adaptation d'un terme au niveau de langue requis ou le choix dominant de la parataxe ${ }^{(13)}$ sont plus difficiles à envisager de façon normée.

Sur la copie A20 La lettre d'Antigone, les annotations marginales, intégralement reproduites ci-dessous, renvoient à cette unique posture.

La posture de " gardien du code», majoritaire dans notre corpus, parfois exclusive, est sans doute considérée comme un impératif professionnel. Il s'agit d'une posture que de nombreux correcteurs adoptent de manière « obligatoire » mais dont la mise en œuvre, en particulier sur des travaux très déficients de ce point de vue, aboutit à masquer, parfois concrètement par l'abondance des signalements d'erreurs, toute autre réaction de lecture.

Il est surtout remarquable que cette posture reste sans relation directe avec le

(11) Rien ne s'oppose à ce que le même lecteur n'adopte successivement différentes postures.

(12) Mais malgré tout variables : les enseignants prennent-ils en compte par exemple les tolérances orthographiques?

(13) Ce dernier exemple est inspiré par les multiples annotations de correcteurs réclamant 1'explicitation par l'élève des liens entre les énoncés (dans les corrections du texte Le Métro de l'enfer par exemple) et la contestation fréquente de la juxtaposition par un usage jugé abusif de la virgule (en particulier dans La lettre d'Antigone). 


\begin{tabular}{|ll|}
\hline Copie A20 & \\
prisonnière (,)je ne dors plus & Ponctuation \\
je m'en fiche & Md \\
appart & Orth \\
neuveu & Orth \\
réclamants & Orth \\
verres & Orth \\
de laisser pourrir & Mal dit \\
neuveu & \\
le seigneur vous punira & (anach) \\
Lui, aurait eu le cran de vous le pardonner & Md \\
d'odace & Orth \\
& \\
\hline
\end{tabular}

texte lu. Les annotations ne rendent que peu compte de la spécificité du texte et sont autonomisées par rapport à la production de l'élève. Tous les textes peuvent alors être corrigés de la même manière ; la posture du « gardien du code » convient à n'importe quel texte, peu importent son mode de production et sa pertinence.

\section{Le "lecteur naïf"}

Contrairement au précédent, le « lecteur naïf »s'intéresse moins au langage qu'à la représentation du monde. Le professeur commente l'action inventée par l'élève en référence au monde d'aujourd'hui, d'un passé à reproduire, voire d'un futur à inventer. Les lois du réalisme sont convoquées mais la posture naïve oublie que le texte se doit de construire son univers de référence. Les annotations rendent compte de la conformité au « monde ordinaire», sans passer par le détour du vraisemblable et tendent ainsi à accréditer une conception «transparente» du langage ${ }^{(14)}$.

Nous illustrons cette posture par quelques annotations marginales recueillies parmi les corrections du Métro de l'enfer:

\section{Copie M7}

- Le métro passe donc dans la même gare que le train?

- Est-ce un train ou un métro ?

\section{Copie M28}

- Logiquement on n'annonce pas la panne après qu'elle est réparée.

- (À propos de l'arrivée finale à la gare) Est-ce donc possible qu'on y trouve tous ces magasins?

L'implication du lecteur qui adopte cette posture est évidente : un lecteur qui croit, qui veut croire, ou feint de croire en la réalité du référent proposé sans l'envisager explicitement dans sa construction langagière. Dès lors les annotations portent sur l' « histoire » et non sur la «narration », ce qui ne va pas sans difficulté. Certains exemples montrent que le correcteur conteste parfois dans l'histoire racontée des propositions tout à fait véridiques, " incroyables » compte tenu de sa connaissance du réel ${ }^{(15)}$.

(14) On sait combien est difficile pour un jeune scripteur l'apprentissage de la vraisemblance. Sur quelles « lois du monde » repose la fiction? Quel contexte (référentiel) est-il impliqué par les actions rapportées? etc. Autant de questions dont les réponses valident ou non les choix de genre, de personnages, d'actions et de narration qui ont été faits par l'auteur de la fiction.

(15) Plusieurs anecdotes pourraient illustrer les conséquences dommageables d'une posture de lecteur naïf. Celui-ci condamne l' « histoire»sans interroger les éventuelles insuffisances de textualisation du monde de référence. 
Cette posture peut néanmoins avoir un réel intérêt sur le plan pédagogique dans certaines circonstances. Le lecteur, évitant de condamner lourdement la représentation du monde du scripteur, interroge naïvement le texte adoptant une attitude lectorale à finalité éthico-pratique au sens de Bernard Lahire. On peut penser que les questions posées alors sont susceptibles de renvoyer l'élève, non pas à une défaillance en tant que sujet, mais à des préoccupations de scripteur soucieux de se faire mieux comprendre, pour peu que le dispositif didactique prévoie des possibilités d'amender le texte initial.

\section{La posture " stimulus-réponse"}

Le jugement de conformité peut porter sur le respect du code ou sur le respect du monde : on voit que ces postures sont relativement indépendantes de la situation de travail scolaire à l'origine de la production écrite. Il peut aussi prendre comme point de référence le sujet à traiter et les consignes afférentes. La posture «stimulus-réponse » recouvre des comportements de correction fréquents dont l'idéal implicite réside dans une bonne adaptation du texte de l'élève à la commande magistrale $(C f$. ci-dessous trois exemples significatifs empruntés à trois textes corrigés différents.)

\section{Copie A8}

Bon devoir respectant bien les consignes indiquées. Le système énonciatif de la lettre a été bien compris. Bonne organisation (introduction du propos et clôture de la lettre).

\section{Copie M4}

Le mécanisme de la nouvelle n'est pas compris : il fallait inventer une histoire où la chute devait étonner, déstabiliser le lecteur. Où est la surprise créée par la chute ?

\section{Copie J10}

Vous avez respecté les consignes d'écriture, même si votre choix d'un objet familier et anodin s'avère pour le moins original.

Le professeur s'intéresse à la réalisation comme réponse, tout est évalué à l'aune de la commande, du sujet proposé et de son traitement. Le rôle du correcteur est conçu comme technique, non au sens du respect du code général du langage mais du respect de la tâche à accomplir voire du réinvestissement de savoirs requis par la tâche.

Le texte produit est-il adapté à la situation d'écriture, conforme aux attentes du professeur compte tenu des activités pédagogiques menées précédemment ? On pourrait évoquer la prégnance d'un modèle béhavioriste. L'écriture doit rendre visibles point par point les compétences acquises. On voit même que l'originalité, si souvent réclamée dans les productions écrites, pourrait devenir soudain un obstacle à l'appréciation positive du texte ( $C f$. ci-dessus Copie J10).

La posture «stimulus-réponse » traduit une forme particulière d'implication. S'il y a implication effective de l'enseignant lecteur, qui fait l'état des lieux des savoirs et savoir-faire du scripteur à un moment donné des apprentissages, le sujet lecteur apparaît peu. L'omniprésence du professionnel occulte celle de l'individu et la relation « ordinaire » entre scripteur et lecteur pourrait bien en être affectée. On peut en effet se demander si la disparition du sujet lecteur n'entraîne pas defacto celle du sujet scripteur, réduit à sa dimension d'apprenant au sens le plus étroit du terme, l'écriture scolaire se limitant alors à l'acquisition d'habiletés scripturales diverses. 


\section{L'" éditeur"}

La posture d' "éditeur» se distingue assez nettement des précédentes : le propos n'est pas d'émettre un quelconque jugement de conformité sinon peut-être avec les désirs égoïstes du lecteur et ses représentations du bon texte. Cette posture tient compte de la spécificité du texte tant sur le plan des contenus que des choix langagiers et son objectif principal est l'amélioration; le professeur commente, fait des suggestions qui s'appuient sur le « déjà écrit ». Il engage l'élève à revoir des points de détail, à reprendre des passages en expliquant parfois comment faire. Le texte est considéré comme en devenir.

Deux exemples illustreront cette posture : le premier correcteur multiplie les interventions sous la forme d'annotations marginales, le second exprime, dans l'annotation générale, un parti pris d'écriture susceptible selon lui d'améliorer la production.

\section{Copie A2 \\ mon frère erre toujours \\ je m'en fiche. \\ jouer avec votre culpabilité... \\ Polynice est votre neuveu; il n'était pas parfait \\ Pourquoi ne pas prendre cette décision de libérer mon frère \\ Où ? Contradiction \\ $\mathrm{Pb}$ de registre. Antigone est noble \\ Est-ce un argument efficace pour mettre Créon de son côté? \\ Pq changer de temps? \\ $\S$ qui aurait mérité d'être dvpé}

\section{Copie M9}

Ton écriture est agréable mais tu dois mettre en place des transitions : mots de liaison, phrase introductive... (alors, puis, après ma descente du train, sur le quai...)

Les annotations incitent à l'action, en l'occurrence celle d'une révision textuelle. L'enseignant peut exprimer ses demandes de manière injonctive ou interrogative, il envisage la réception de ses annotations dans le cadre d'une communication suivie: il s'inscrit au moins rhétoriquement dans une dynamique de réécriture qui devrait mener à l'examen ultérieur d'un nouvel état du texte.

\section{La posture du " critique"}

Le lecteur se place en position de commentateur d'un texte mis à distance. Le texte est pensé comme une construction; il devient un objet de réflexion à la manière d'un texte littéraire lors d'une lecture analytique même si les annotations semblent se rapprocher de notes de lecture et traduisent une circulation relativement libre de l'ensemble au détail.

Une correction du devoir La lettre d'Antigone en offre un exemple représentatif:

\section{Copie A18}

C'est à peine si j'ai une âme ici, mais

être en cage m'importe peu, ce qui me met dans cet état, "grand "Créon, nous sommes du même sang, je me dois de le faire rejoindre les siens. Que son roi autrefois n'était qu'un lâche qui a dit " oui »...

Vous ne vous préoccupez donc pas de ce que dira le monde? et le seigneur vous punira:

Vous, vous mourrez fièrement, comme un roi ayant bien servi son peuple, lui, le méritait aussi!

Jolie formule

« grand » ironie : bien

Héroïne tragique, honneur B

Contradiction // fin de la lettre Pas très antique

Contradiction : « vous ne vous préoccupez pas de ce que dira le monde?" 
Les correcteurs qui adoptent durablement cette posture sont rares dans notre corpus. Elle apparaît lorsque le devoir est jugé de qualité et qu'une possible connivence avec le scripteur est postulée par l'enseignant. Ce dernier fait état de réactions de lecteur en se situant par rapport à des choix dont il estime le scripteur capable et émet des jugements de nature esthétique ou stylistique. Les propos sont parfois teintés d'humour et les désapprobations sont souvent à saisir sous la forme d'interrogations.

\section{La correction : une pratique à éclairer}

Le lecteur de copies affirme son appartenance à la communauté des enseignants correcteurs par des traces caractéristiques et des modalités d'inscription relativement conventionnelles. Chacun est bien persuadé qu'il est en mesure de faire le départ entre ce qui est bon et ce qui l'est moins et de produire un métadiscours pertinent. On voit pourtant que l'analyse de la correction de copies fait émerger nombre de problèmes qui vont bien au-delà de la simple recherche d'objectivité ou de justesse. Elle interroge la discipline dans son ensemble autant que le geste professionnel proprement dit. Il serait dommageable que la sophistication des procédures d'évaluation masque la réalité des rapports qui se construisent dans la lecture des travaux d'élèves comme dans toute autre lecture. La réflexion sur les postures de lecture développées spontanément par les enseignants peut alors constituer une ressource utile.

\section{Après la « correction ": le « corrigé »?}

Toute lecture suscitant une attente, on peut considérer que la situation de correction tend à actualiser cette attente sous la forme non pas d'un corrigé type mais d'un texte « virtuel », étroitement dépendant des propositions de l'écrit en cours de correction et qui, aux yeux du professeur dont il constituerait la meilleure version possible, donnerait toute satisfaction. Pour mieux comprendre l'activité corrective, il était nécessaire de rendre visible ce texte fantôme et de le confronter au métadiscours de correction. Si des dysfonctionnements ont été notés par les correcteurs, on peut penser que ceux-ci sont en mesure de proposer une version plus conforme à leurs attentes. C'est l'origine de la contrainte qui a été imposée aux correcteurs du Métro de l'enfer : produire une réécriture du texte de l'élève qui éviterait les dysfonctionnements initialement repérés, autrement dit produire une version du texte qui soit en mesure d'échapper aux critiques de la correction ${ }^{(16)}$. L'étude et la comparaison d'une trentaine de réécritures obtenues à partir du texte source permet de tirer quelques enseignements.

\section{Quelques caractéristiques des réécritures}

L'examen des réécritures nous informe des caractéristiques dominantes du «bon» texte selon les professeurs de notre échantillon. Si le contenu sémantique du Métro de l'enfer est accepté, de nombreuses réécritures font apparaître des choix que nous pouvons confronter aux interventions correctives. Le texte de l'élève entre parfois en conflit avec une représentation précise du texte apprécié dans le cadre scolaire et dont les réécritures portent le témoignage. Nous en donnerons quelques exemples.

(16) Une heure environ a été accordée aux re-scripteurs pour produire, en gardant comme point de départ le texte de l'élève, une version, sinon parfaite, du moins plus conforme à ce qu'ils considéraient comme un «bon » texte. 
À la succession de paragraphes brefs, les enseignants préfèrent une mise en page plus dense où les paragraphes constituent des unités d'une certaine ampleur, conditionnées par la progression du récit et majoritairement redondantes par rapport au balisage temporel. La réécriture 20 que nous reproduisons partiellement en donne un exemple particulièrement caricatural qui correspond assez bien à la prise en compte des reproches de correcteurs réclamant, ou une meilleure mise en paragraphes, ou l'introduction de marques de cohésion.

\section{Réécriture M20}

Cela faisait déjà plusieurs minutes que j'attendais le train, debout, immobile, au milieu d'une foule à la fois dense et oppressante. [...]

Une fois le train immobilisé, la masse des voyageurs [...] Une fois à l'intérieur je fus confronté $[. .$.$] Le train démarra enfin.$

Cependant au bout de dix minutes, alors que le train s'engageait dans un tunnel sombre et triste $[\ldots]$

Pourtant quelques minutes plus tard le train de l'enfer [...]

$\mathrm{Au}$ bout d'une demi-heure, le train entrait en gare [...]

Une conception très classique du paragraphe, sans doute attachée au modèle canonique du récit, s'exprime ici. Cet apprentissage semble diriger à la fois les corrections et les réécritures; adopter une superstructure narrative efficace ne suffit pas à produire un récit réussi, il faut de surcroît que ce dernier exhibe ses caractéristiques de manière explicite. Les marqueurs temporels et spatiaux en sont des éléments clefs. Le récit, même complexe, n'échappe pas à un certain conformisme. C'est le déficit de stéréotypie de la production scolaire qui semble soumis d'abord à correction.

La reprise des étapes du récit est le fait de tous mais les modifications internes sont fréquentes. Les variations temporelles introduites tendent à revoir le rapport premier plan / arrière plan de l'élève et les enseignants adoptent finalement une écriture beaucoup plus traditionnelle. Le modèle littéraire sous-jacent est sans doute à rechercher davantage chez les nouvellistes du XIX ${ }^{\mathrm{e}}$ siècle, ou tout au moins dans ce que l'enseignement secondaire en retient comme traits saillants, que dans une écriture plus contemporaine.

On pouvait aussi penser que les passages épargnés par la critique lors de la correction seraient repris sans modifications. Ils sont rares, les re-scripteurs ne reprennent généralement pas les phrases de l'élève à l'identique. Bien que les reprises lexicales soient abondantes, la syntaxe de l'élève est fortement rejetée : les professeurs répugnent en général à introduire des réécritures pointillistes qui correspondraient pourtant aux traces de corrections, souvent de faible empan. Autrement dit, quand le correcteur souligne ou annote un mot, il réécrit une phrase !

Les propositions de réécritures ont pour point commun l'évitement de tout traitement créatif de l'ambiguïté. Le traitement normatif de l'énonciation n'engendre pas de recherche autre qu'une homogénéisation minimale. L'éventuelle hésitation sur le moyen de transport ne laisse place à aucune interrogation.

Le choix du « modèle de train », un train à vapeur, qui n'est pas spécifique des enseignants puisque l'élève y a aussi recours, mérite qu'on s'y arrête. La connaissance du topos de la gare et du train fonctionne ici efficacement mais ne déclenche pas l'emploi, pour son évocation, du vocabulaire spécifique qui pouvait participer à la vraisemblabilisation du récit ; la commande faite aux élèves d'être plus exigeant quant au choix des mots, fréquemment attestée chez les enseignants, n'est pas réellement mise en œuvre lorsque ceux-ci sont mis en situation d'écriture. Le réalisme 
convoqué est un réalisme minimaliste, non pas celui qui permet de faire voir mais celui qui se contente d'éviter les contradictions qu'un correcteur pourrait sanctionner. Certains ajouts et remplacements lexicaux vont dans ce même sens. Pour obéir aux condamnations de termes jugés inadéquats, les re-scripteurs utilisent parfois la substitution synonymique mais, le plus souvent, les réécritures sont significativement différentes et marquées par une propension à l'emploi de stéréotypes d'expression. C'est davantage l'emploi de l'expression «toute faite » reconnue comme stéréotype qui est recherchée plutôt que le mot dans sa précision dénotative.

Compte tenu de la brièveté relative du texte, on note peu de suppressions mais des remplacements fréquents. L'ajout est aussi très important : il laisse à penser que les re-scripteurs cherchent à compléter les places vacantes offertes par la présence de groupes syntaxiques peu expansés. Les termes choisis semblent s'appeler sous forme de séries lexicales. On peut penser à une recherche de saturation de l'expression qui tient lieu d'amélioration ${ }^{(17)}$. À la densité recherchée du point de vue de l'aspect général du texte s'ajoute donc une densité interne. Celle-ci ne va pas nécessairement de pair avec le respect de la syntaxe originale. Si l'examen détaillé des réécritures nous invite à constater le respect du propos initial, le résultat est souvent marqué par une simplification des structures syntaxiques de l'élève.

Nous pouvons interpréter les modifications introduites comme relevant d'un parti pris stylistique : le « devoir » apparaît comme un lieu de mise en application d'un ensemble de préceptes qui fondent une esthétique proprement scolaire de l'écrit.

\section{Conclusion}

Le métier d'enseignant s'appuie sur un ensemble de connaissances, de savoirfaire, de gestes professionnels qui ont acquis une telle force d'évidence qu'il n'est pas toujours facile d'en faire des objets de questionnements. Il nous est apparu rapidement qu'à la complexité de l'objet, le texte de l'élève, répondent la complexité de la tâche à accomplir par le professeur et la complexité du correcteur lui-même.

L'examen de la situation de communication qui met en présence l'élève et l'enseignant montre que le dialogue qui se construit à l'occasion de la correction est difficile et demeure peu étudié alors que l'on constate aisément, à la lecture des traces laissées sur les copies, que le professeur engage dans sa lecture toute sa subjectivité de lecteur. Celle-ci est peut-être d'autant plus sollicitée que nous avons choisi de limiter nos investigations à des écrits fictionnels, pour lesquels la référence littéraire est omniprésente et en relation permanente avec les activités de lecture. Une « subjectivité complexe ", si l'on peut dire, où l'expression du professeur en tant que lecteur est parasitée par les déterminations qui pèsent sur lui en tant que correcteur. À une lecture libre, une lecture « ordinaire», se superpose les contraintes d'une «lecture de correcteur », dont l'essentiel de l'activité réside dans l'identification des dysfonctionnements du texte qu'il construit parfois autant qu'il les découvre.

Ces deux lectures, ne sont pas totalement étrangères l'une à l'autre. Il s'agit bien dans les deux cas de construire du sens ; mais, quand la première se confronte aux propositions du texte légitime, la seconde pose, de manière axiomatique, la nature contestable de la production d'élève. Cette dernière tend alors à être appréhendée comme la version dégradée d'un texte virtuel qui n'existe que dans l'imaginaire du correcteur singulier. On comprend peut-être alors mieux pourquoi les annotations

(17) On doit ajouter que la recherche de saturation est appliquée presque uniquement par recours au vocabulaire des sensations et des sentiments, les éléments proprement descriptifs sont peu convoqués sinon dans la scène finale. 
correctives se focalisent sur des « erreurs » ou des « mauvais choix » dont l'identification n'est pas toujours partagée.

La soumission, dans une forme standardisée par la tradition, de la présentation des annotations n'empêche pas la manifestation d'écarts importants d'un correcteur à l'autre. On peut y découvrir le caractère éclaté et disparate des savoirs de référence des enseignants. L' « épreuve » de la correction oblige à réinterroger la validité de notions disciplinaires qu'un discours plus distancié n'aurait vraisemblablement pas mentionné. Il faut y ajouter d'autres questions concernant tant la cohérence des choix pédagogiques et de l'apprentissage de l'écriture que la conception du texte qui s'y exprime.

C'est vraisemblablement la conception du texte véhiculée par le discours des correcteurs qui apparaît comme une réalité particulièrement remarquable. Qu'il s'agisse des expérimentations centrées sur la «correction» de copies ou de celle qui donnait aux professeurs l'occasion d'actualiser « un texte attendu » le constat est identique. La correction de copies, assise sur une tradition de solitude et d'invisibilité, est le lieu de tous les excès en matière de repli normatif.

Le lecteur, spécialiste de la discipline et du texte littéraire, tend à disparaître quand la posture du correcteur s'affirme. Les interventions que nous avons analysées montrent clairement la prégnance d'une « dimension technicienne » de la correction, centrée avant tout sur la mise en conformité du texte avec les normes, en particulier grammaticales et orthographiques, qui peuvent aller jusqu'à occulter tout autre regard porté sur la production; le caractère dialogique de la correction risque bien d'en être dramatiquement affecté, d'autant qu'une production écrite, quand elle est perçue comme qualitativement inférieure, accentue la tendance des correcteurs au repérage d'erreurs ponctuelles centrées sur le code.

L'ensemble de ces résultats incite à penser que la tension qui existe entre «posture de lecteur» et « posture de correcteur» se résout le plus souvent au profit de cette dernière dans son acception la plus étroite. D'autres points méritent aussi d'être soulignés.

Malgré le rapport de proximité avec les textes littéraires, que la logique de lecture-écriture en vigueur dans l'enseignement tend à instituer, l'espace de la production écrite semble incompatible avec l'expression de la complexité, ce qui constitue un réel paradoxe. Face à un dysfonctionnement textuel identifié, c'est majoritairement une demande de «simplification » qu'exprime le correcteur. Simplification de la structure, présentation canonique des références spatio-temporelles, homogénéisation des structures syntaxiques, valorisation de l'explicite apparaissent comme des moyens sûrs de proposer un écrit recevable. La tendance de l'univers scolaire à instituer un cadre normatif parfois beaucoup plus contraignant que celui que mettent en œuvre les pratiques sociales de référence, à construire des hypernormes, s'exprime ici de manière évidente dans les champs les plus divers.

On a pu par ailleurs constater que la valorisation systématique de ce que nous pouvons nommer, à la suite de J.-L. Dufays, le stéréotype était une constante des professeurs mis en situation de correction, et on doit ajouter qu'elle occupe aussi une place de choix dans les réécritures que nous avons analysées. Si la cohérence entre les comportements correctifs et les choix scripturaux des enseignants est assurée, elle ne manque pas néanmoins de susciter des questions plus fondamentales.

La qualité d'une production écrite scolaire est-elle relative à la simplicité des moyens mis en œuvre? La pratique de l'écriture fictionnelle a-t-elle pour fonction essentielle l'acquisition de stéréotypes?

Il ne faudrait pas ici donner l'idée qu'un refus de la norme ou du stéréotype pourrait tenir lieu de principe didactique. Ceux-ci sont indispensables à la construction 
d'un consensus langagier, social et identitaire que l'école doit garantir, mais il semble toujours dangereux de naturaliser des réalités culturelles ou des fonctionnements langagiers. On peut craindre qu'une soumission à la stéréotypie qui n'en construit pas la conscience n' aboutisse à des postures de lecteur et de scripteur stérilisantes. Si l'acquisition des stéréotypes est nécessaire, ne peut-elle s'inscrire dans une posture dialectique et critique qui permette d'apprendre à les utiliser en questionnant leur construction, en percevant leurs intérêts et leurs limites, en comprenant leur renouvellement pour éviter le figement de l'expression qui peut rapidement confiner au figement de la pensée.

C'est en ce sens qu'il a paru important de faire émerger, à partir des conduites singulières de correction, des modes d'intervention qui, même s'ils sont plus rares dans les corpus analysés, peuvent être construits en «postures de correcteur » diversifiées susceptibles de permettre une approche plurielle de l'activité de correction.

Le recours à la notion de « postures de correcteur» peut ainsi participer au développement d'une prise de conscience éclairée des comportements spontanés du correcteur empirique et susciter une diversification assumée des pratiques de correction mieux à même de répondre aux besoins et d'assurer la richesse et l'efficacité du dialogue entre l'élève et son lecteur.

\section{Eléments bibliographiques}

BARRÉ-DE MINIAC, C. (2000) : Le rapport à l'écriture-aspects théoriques et didactiques, collection «Les Savoirs mieux », Villeneuve d'Ascq, Presses du Septentrion.

BARRÈRE, A. (2003) : "Les élèves face à l'évaluation : de l'imprévisibilité à l'opacité », Université de Lille 3 - PROFEOR, in Recherches $\mathrm{n}^{\circ} 38$.

BAYARD, P. (2000) : Comment améliorer les æuvres ratées?, Paris, Éditions de Minuit.

Bessonnat, D. (2000) : «Une année de réécriture en $3^{\text {ème }}$ », in Pratiques n ${ }^{\circ}$ 105/106, juin.

BISHOP, M.-F. (2005) : «Les annotations, indicateurs des fonctions de la rédaction : parcours historique », in Repères, $\mathrm{n}^{\circ} 31$, INRP.

BUCHETON, D. (2000) : «Les postures de lecture des élèves au collège » in Lecture privée et lecture scolaire, La question de la littérature à l'école, CRDP de Grenoble, 1999, puis in Enseigner la littérature, Delagrave et CRDP Midi-Pyrénées.

CHABANNE, J.-C. (2002) : Écrire en ZEP, un autre regard sur les écrits des élèves, Delagrave.

Chardenet, P. (2003) : «La correction : notion et pratiques », in Le Français aujourd'hui, Gestes et enjeux de la correction, $\mathrm{n}^{\circ} 140$, janvier. 
CHARlot, B., BAUtiER, É. et RocheX, Y. (1992) : École et savoir dans les banlieues et ailleurs, Armand Colin.

Charolles, M. (1989) : Pour une didactique de l'écriture, Université de Metz, Centre d'Analyse Syntaxique.

CombetteS, B. (1992) : L'organisation du texte, Centre d'analyse syntaxique de l'Université de Metz, Collection Didactique des textes.

Compagnon, A. (1998) : Le démon de la théorie, Littérature et sens commun, Paris, Edition du Seuil.

DABÈNE, M. (1987): L'adulte et l'écriture, contribution à une didactique de l'écrit en langue maternelle, Bruxelles/Paris, De Boeck.

DAUNAY, B. (2007) : «Etat des lieux en didactique de la littérature », Revue française de pédagogie, $\mathrm{n}^{\circ} 159$.

— (2007) : «Comment la recherche didactique évalue les pratiques d'enseignement de la littérature ", in Enseigner et apprendre la littérature aujourd'hui, pour quoi faire?, J.-L. Dufays éditeur, P. U. de Louvain.

DELFORCE, B. (1986) : «Évaluer les écrits : correction de copies production de textes démarches d'apprentissage », Bulletin du CERTEIC, Lille, $\mathrm{n}^{\circ}$ 7, 3 février.

DEMOUGIN, P. (2004) : «Le lecteur et sa parole : traces écrites d'une parole recomposée dans l'acte de lecture ", Le sujet lecteur, lecture subjective et enseignement de la littérature, Actes du colloque « Sujets lecteurs et enseignement de la littérature » sous la direction de A. Rouxel et G. Langlade, Presses Universitaires de Rennes.

DOQUET-L ACOSTE, C. (2003) : Étude génétique de l'écriture sur traitement de texte d'élèves de Cours Moyen 2, année 1995-96, Thèse de Doctorat de l'Université de Paris III, sous la direction de Jacqueline Authier-Revuz.

DUfAYS, J.-L. (1994) : Stéréotype et lecture, Liège, Mardaga.

- (2007) : Enseigner et apprendre la littérature aujourd' hui, pour quoi faire?, Actes des rencontres des chercheurs en didactique de la littérature, Louvain-laNeuve, mars, J.-L. Dufays éditeur, Presses Universitaires de Louvain.

DUMORTIER, J.-L. (2001) : Lire le récit de fiction, pour étayer un apprentissage : théorie et pratique, Bruxelles, Editions De Boeck Duculot.

ECO, U. (1979) : Lector in fabula, Paris, Grasset.

- (1992) : Les limites de l'interprétation, Grasset.

EVA [groupe] (1991) : Évaluer les écrits à l'école primaire, Cycles II \& III., INRP, Hachette Éducation.

FABRE, C. (1990) : Les Brouillons d'écoliers ou l'entrée dans l'écriture, Grenoble, Ceditel / L'atelier du texte.

— (1994) : «Ce qui vaut pour un manuscrit d'écrivain vaut-il pour un brouillon d'écolier? ", Le Français aujourd'hui, n 108.

FAYOL, M. (1997) : Des idées au texte, Psychologie cognitive de la production verbale, orale et écrite, Presses Universitaires de France, Le psychologue, Paris.

FourtanieR, M.-J., LANGLADE, G. (2000) : (présentation) « Enseigner la littérature », Actes du colloque Enjeux didactiques des théories du texte dans l'enseignement du français, Delagrave, CRDP Midi-Pyrénées.

Garcia-Debanc, C. (1995) : «Le lire dans 1'écrire », in Pratiques, n86, juin.

GARCIA-DEBANC, C., FAYOL, M. (2002) : «Apports et limites des modèles du processus rédactionnel pour la didactique de la production écrite. Dialogue entre psycholinguistes et didacticiens ", in Pratiques 115/116.

Genette, G. (1991) : Fiction et diction, Paris, Seuil. 
HALTÉ, J.-F. (1984) : «L'annotation de copies, variété ou base du dialogue pédagogique », in Pratiques, ${ }^{\circ} 44$.

— (2002) : «Didactique de l'écriture, didactique du français, vers la cohérence configurationnelle », in Pratiques n¹15/116.

HAY, L. (1985) : «Le texte n'existe pas. Réflexions sur la critique génétique », Poétique, $\mathrm{n}^{\circ} 62$, Seuil.

Jorro, A. (2002) : Professionnaliser le métier d'enseignant, Paris, E.S.F.

Kerbrat-Orecchioni, C. (1980) : L'énonciation, De la subjectivité dans le langage, Armand Colin.

KERVYN, B. \& DUFAYS, J.-L. (2003) : «Quels usages du stéréotype dans l'écriture au secondaire ? Observations et propositions. " Pratiques, n ${ }^{\circ} 11-118$.

LAFONT TERRANOVA, J. et COLIN, D. (2002) : «Les enseignants de collège et l'écriture, des déclarations aux représentations », in Pratiques $\mathrm{n}^{\circ} 115 / 116$.

LAHIRE, B. (1993): «Lectures populaires : les modes d'appropriation des textes », in Revue française de pédagogie, ${ }^{\circ} 104$, I.N.R.P., sept.-oct.-nov.

- (1998) : L'homme pluriel, les ressorts de l'action, Paris, Nathan.

LANGLADE, G. (2005) : «L'activité fictionnalisante du lecteur », CELAM, Université de Rennes II.

Lumbroso, O. (2007) : «Esquisse d'un dialogue entre didactique de l'écrit et critique génétique : l'élève "auteur-dessinateur » in Revue Française de Pédagogie, $\mathrm{n}^{\circ} 159$.

Molinier, G. et Viala, A. (1993) : Approches de la réception, Presses Universitaires de France.

ORIOL-BOYER, C. (1990) : «La réécriture », in La réécriture, Actes de l'Université d'été de Cerisy-la-Salle (1988), sous la direction de Claudette Oriol-Boyer, Édition CEDITEL, Université de Grenoble Stendhal.

PLANE, S. (2000) : «Éléments pour un usage didactique du traitement de texte. Écrire, réécrire et réviser sur ordinateur. ", in Pratiques n¹05/106, juin.

REICHLER-BÉGUELIN, M.-J. (1992) : «L'approche des "anomalies” argumentatives », Pratiques $\mathrm{n}^{\circ} 73$, mars.

REUTER, Y. (2004) : «Définition, statut et valeurs des dysfonctionnements en didactique », in Repères, $\mathrm{n}^{\circ} 31$.

ROUXEL, A. (2004) : «Autobiographies de lecteurs à l'entrée au lycée » in Le Français aujourd'hui, $\mathrm{n}^{\circ} 147$, octobre.

RouXEL, A. et LANGLADE, G. (dir.) (2004) : Le sujet lecteur, lecture subjective et enseignement de la littérature, Actes du colloque « Sujets lecteurs et enseignement de la littérature », Presses Universitaires de Rennes.

TAUVERON, C. (2007) : « Le texte singulier de l'élève ou la question du sujet scripteur », in Le Français aujourd'hui, n 157.

TAUVERON, C. et SÈVE, P. (2005) : Vers une écriture littéraire ou comment construire une posture d'auteur à l'école, Hatier.

Tochon, F.-V. (1993) : L'Enseignant-expert, Nathan Pédagogie. 